\title{
Global Stability for Holomorphic Foliations in Kaehler Manifolds
}

\author{
Jorge Vitório Pereira ${ }^{1}$
}

\begin{abstract}
We prove the following theorem for Holomorphic Foliations in compact complex kaehler manifolds: if there is a compact leaf with finite holonomy, then every leaf is compact with finite holonomy. As corollary we reobtain stability theorems for compact foliations in Kaehler manifolds of Edwards-Millett-Sullivan and Hollman.
\end{abstract}

\section{Introduction}

The question of global stability is recurrent in the theory of foliations. The work of Ehresmann and Reeb establishes the so called global stability theorem, which says that if $\mathcal{F}$ is a transversely orientable codimension one foliation in a compact connected manifold $M$ that has a compact leaf $L$ with finite fundamental group, then every leaf of $L$ is compact with finite holonomy group[Ca-LN]. Counterexamples for codimension greater than one are known since the birth of the theorem. Here we want to abolish the hypothesis on the codimension for a special kind of foliation, namely holomorphic foliations in complex Kaehler manifolds. In other words we are going to prove the following :

Theorem 1. Let $\mathcal{F}$ be a holomorphic foliation of codimension $q$ in a compact complex Kaehler manifold. If $\mathcal{F}$ has a compact leaf with finite holonomy group then every leaf of $\mathcal{F}$ is compact with finite holonomy group.

Another kind of stability problem was posed by Reeb and Haefliger. The question was the stability of compact foliations, that is, if a foliation has all leaves compact is the leaf space Hausdorff? Positive answers to this problem arose in the work of Epstein[Ep], Edwards-Millet-Sullivan[EMS], Holmann[Ho], etc. There are plenty situations where the leaf space is not Hausdorff. Sullivan found a example in the $C^{\infty}$ case [Su], Thurston in the analytic case[Su] and Müller in the holomorphic case[Ho]. The examples of Sullivan and Thurston live in compact manifolds, and Müller's in a non-compact non-Kaehler manifold. As corollary of the theorem we reobtain Holmann's result and a special case of [EMS]'s outstanding Theorem.

Corollary. [EMS,Ho] Suppose $M$ is a complex Kaehler manifold. If $\mathcal{F}$ is a compact foliation, i.e., every leaf is compact, then every leaf has finite holonomy group. Consequently, there is an upper bound on the volume of the leaves, and the leaf space is Hausdorff.

The author would like to thanks B. Scárdua for valuable conversations.

\footnotetext{
${ }^{1}$ Supported by IMPA-CNPq
} 


\section{The Leaf Volume Function}

Let $\mathcal{F}$ be a holomorphic foliation of a complex Kaehler manifold $(M, \omega)$. As in $[\mathrm{Br}]$ we define

$$
\Omega=\left\{p \in M \mid \text { the leaf } L_{p} \text { through } \mathrm{p} \text { is compact with finite holonomy }\right\}
$$

By the local stability theorem of Reeb[Ca-LN] $\Omega$ is an open set of $M$. Set, for every $p \in \Omega, n(p) \in \mathbb{N}$ to be the cardinality of the holonomy group of $L_{p}$. If $d$ is the dimension of the leaves then we define volume function of $\mathcal{F}$ :

$$
T: \Omega \longrightarrow \mathbb{R}^{+}, T(p)=n(p) \int_{L_{p}} \omega^{d}
$$

Lemma 1. $T$ is a continuous locally constant function in $\Omega$.

proof. The continuity is obvious. We have to prove that $T$ is locally constant. To do this we have just to observe that it is constant in the residual subset of $\Omega$, formed by the union of leaves without holonomy[G,p. 96]. By the Reeb local stability theorem there is a saturated neighborhood for each leaf in this set where all leaves are homologous. Then using the closedness of $\omega^{d}$ and Stokes Theorem we prove the lemma.

Remark - In fact, the proof of this lemma is essentially already contained in [Ho].

\section{A Lemma about $\operatorname{Diff}\left(\mathbb{C}^{n}, 0\right)$}

In 1905 Burnside[Bu] proved that if $G$ is a subgroup of $G L(n, F)$, where $F$ is a field of characteristic zero, with exponent $e$, then $G$ is finite with $\operatorname{cardinality}(G) \leq e^{n^{3}}$. Recalling that a group has exponent $e$ if every element $g$ belonging to the group is such that $g^{e}=1$. From the generalization of this result by Herzog-Praeger[HP] we obtain :

Lemma 2. If $G$ is a subgroup of $\operatorname{Dif} f\left(\mathbb{C}^{n}, 0\right)$ with exponent e then $G$ is finite with $\operatorname{cardinality}(G) \leq e^{n}$.

proof. If for each element of $G$ we consider its derivative we obtain a subgroup of $G L(n, \mathbb{C})$ with exponent $e$. Thus we only have to prove that the normal subgroup $G_{0}$ of $G$, formed by its elements tangent to the identity is the trivial group.

Let $g \in G_{0}$, then $g^{e}=I d$. Defining $H(x)=\sum_{i=1}^{e} D g(0)^{-i} g^{i}(x)$, we see that :

$$
H \circ g(x)=D g(0) D g(0)^{-1} \sum_{i=1}^{e} D g(0)^{-i} g^{i+1}(x)=D g(0) H(x)
$$

Hence $\mathrm{g}$ is conjugated to its linear part, and therefore g must be the identity. 


\section{Proof of the Theorem 1}

Let $\mathcal{F}$ be as in the theorem. Consider the connected component of $\Omega$ containing the leaf $L$ that is compact and with finite holonomy, and call it $\Omega_{L}$. The volume function $T$ is constant in $\Omega_{L}$ by Lemma 1 , so if $p \in \partial \Omega_{L}$ we have that the leaf through $p$ is aproximated by leaves with uniformly bounded volume, so it has bounded volume and is compact(here we use the fact that the manifold is compact to achieve the compactness of the leaf). The holonomy group of $\Omega_{L}$ has finite exponent, because for any transversal $\Sigma$ of $L_{p}, \Sigma \cap \Omega_{L}$ will be an open set such that every leaf of $\Omega_{L}$ cuts it in at most $m$ points. Thus for every holomy germ $h$ of $L_{p},\left(h^{m !}\right)_{\mid \Sigma \cap \Omega_{L}}=I d$.

Analytic continuation implies that $h^{m !}=I d$. Using Lemma 2, we see that $\partial \Omega_{L}=\emptyset$, and prove the theorem.

The Corollary follows observing that the set of leaves without holonomy is residual and that we don't need the compactness of the manifold to assure that a limit leaf is compact. Then the holonomy group of each leaf is finite and by the results of Epstein[Ep] we get the consequences.

Remark - The same proof works in a more general context. We have just to suppose that our foliation is transversely quasi-analytic and that there is a closed form which is positive on the (n-q)-planes of the distribution associated to the foliation.

\section{References}

[Br] Brunella, M. :A global stability theorem for transversely holomorphic foliations, Annals of Global Analysis and Geometry 15(1997), 179-186

[Bu] Burnside, W. : Proc. London Math. Soc. (2) 3 (1905), 435-440

[Ca-LN] Camacho, C. and Lins Neto, A. : Geometric theory of foliations, Birkhauser, 1985

[EMS] Edwards, R., Millett K. and Sullivan D. : Foliations with all leaves compact, Topology 16(1977), 13-32

[Ep] Epstein, D.B.A. : Foliations with all leaves compact, Ann. Inst. Fourier 26, 1(1976), 265-282

[G] Godbillon, C. : Feuilletages, études géométriques, Birkhäuser, Basel, 1991

[HP] Herzog M. and Praeger C. : On the order of linear groups with fixed finite exponent, Jr. of Algebra 43(1976), 216-220

[Ho] Holmann, H. : On the stability of holomorphic foliations, LNM 798(1980), 192-202

[Su] Sullivan, Dennis : A counterexample to the periodic orbit conjecture, Inst. Hautes Études Sci. Publ. Math. 46(1976),5-14

Jorge Vitório Bacellar dos Santos Pereira

Email : jvp@impa.br

Instituto de Matemtica Pura e Aplicada, IMPA

Estrada Dona Castorina, 110 - Jardim Botnico

22460-320 - Rio de Janeiro, RJ, Brasil 